\title{
COMET/ASTEROID PROTECTION SYSTEM (CAPS): A SPACE-BASED SYSTEM CONCEPT FOR REVOLUTIONIZING EARTH PROTECTION AND UTILIZATION OF NEAR-EARTH OBJECTS
}

\author{
Daniel D. Mazanek, Carlos M. Roithmayr, and Jeffrey Antol

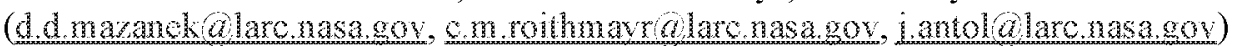 \\ Spacecraft and Sensors Branch, ASCAC \\ NASA Langley Research Center \\ Hampton, Virginia USA \\ Linda Kay-Bunnell and Martin R. Werner

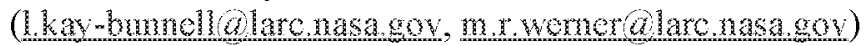 \\ Joint Institute for Advancement of Flight Sciences (JIAFS) \\ The George Washington University \\ Hampton, Virginia USA \\ Sang-Young Park \\ (s.jarkolarenasagov) \\ Swales Aerospace, Inc. \\ Hampton, Virginia USA \\ Renjith R. Kumar \\ (renilorama-3ne com) \\ Analytical Mechanics Associates, Inc. \\ Hampton, Virginia USA
}

\begin{abstract}
$\underline{\text { ABSTRACT }}$
There exists an infrequent, but significant hazard to life and property due to impacting asteroids and comets. There is currently no specific search for long-period comets, smaller near-Earth asteroids, or smaller shortperiod comets. These objects represent a threat with potentially little or no warning time using conventional ground-based telescopes. These planetary bodies also represent a significant resource for commercial exploitation, long-term sustained space exploration, and scientific research. The Comet/Asteroid Protection System (CAPS) would expand the current detection effort to include long-period comets, as well as small asteroids and short-period comets capable of regional destruction. A space-based detection system, despite being more costly and complex than Earth-based initiatives, is the most promising way of expanding the range of detectable objects, and surveying the entire celestial sky on a regular basis. CAPS is a future spacebased system concept that provides permanent, continuous asteroid and comet monitoring, and rapid, controlled modification of the orbital trajectories of selected bodies. CAPS would provide an orbit modification system capable of diverting kilometer class objects, and modifying the orbits of smaller asteroids for impact defense and resource utilization. This paper provides a summary of CAPS and discusses several key areas and technologies that are being investigated.
\end{abstract}

Copyright (C) 2002 by the American Institute of Aeronautics and Astronautics, Inc. No copyright is asserted in the United States under Title 17, US Code. The US Government has a royalty-free license to exercise all rights under the copyright claimed herein for Governmental Purposes. All other rights are reserved by the copyright owner. 
$5^{\text {rd }}$ International Astronautical Congress IAC-02-IAA.13.4./Q.5.1.01

\section{INTRODUCTION}

An enormous number of asteroids and comets orbit the Sun, ranging in size from pebbles to mountains. Fortunately, only a tiny number of these objects cross the Earth's orbit, and our atmosphere protects us from small and structurally weak objects. Impacts are extremely infrequent events relative to a human lifetime, but have the potential for massive loss of life and property. Impacts have occurred in the past and will occur in the future. The energy released from an impactor capable of causing surface damage ranges from $\sim 10$ megatons (MT) of TNT to billions of megatons ( $1 \mathrm{MT}=4.185 \times 10^{15}$ Joules $)$. A $10 \mathrm{MT}$ impact can result from an object approximately $50 \mathrm{~m}$ in diameter, and is roughly equal to 700 Hiroshima size explosions. This class of impact is estimated to occur every several hundred years (or possibly less) and can cause regional destruction. An impact with a $1 \mathrm{~km}$ diameter object, capable of releasing roughly $100,000 \mathrm{MT}$ and resulting in a global catastrophe, can be expected to occur every several hundred thousand years to a million years. An impact from a $10 \mathrm{~km}$ object, like the one believed to have caused the great dinosaur extinction 65 million years ago, can be expected on an interval of 10 million years or greater.

Earth approaching asteroids and comets are collectively termed NEOs (near-Earth objects). The goal of current search efforts is to catalog and characterize by 2008 the orbits of $90 \%$ of the nearEarth asteroids (NEAs) larger than $1 \mathrm{~km}$ in diameter, currently estimated to number between 900 and 1300. Devastating impacts can also occur from smaller NEAs, short-period comets (SPCs) in asteroid-like orbits, and long-period comets (LPCs) which do not regularly enter near-Earth space since their orbital periods range from 200 years to million of years.

The Comet/Asteroid Protection System (CAPS) is a future space-based system concept ( 25 or more years from now) designed to detect and mitigate the entire range of threatening comets and asteroids. The initial focus is to determine the feasibility of protecting against $1 \mathrm{~km}$ class long-period comets, including inactive nuclei. The system is designed also to protect against smaller LPCs, as well as NEAs and SPCs capable of regional destruction.
October 10-19, 2002

Houston, Texas USA

Although the primary motivation for CAPS is to provide protection against impacting comets and asteroids, it is anticipated that the system and technologies developed would have many additional benefits extending to governments (U.S. and international), the commercial sector, the scientific community, and academia. The CAPS detection system would provide an astronomical asset that could observe extremely faint or small targets (both planetary bodies and extra-solar objects), providing an unprecedented level of scientific observations while surveying the entire celestial sky on a regular basis. The CAPS orbit modification system could enable exploitation of the vast economic resources available from NEOs, and promote synergistic technologies for other future space missions. Technologies that will permit the future exploration and colonization of the solar system (e.g., high power and thermal management systems, high thrust and specific impulse propulsion, and power beaming) are applicable also to the mitigation of Earth impacting comets and asteroids. Additionally, there is tremendous benefit in "practicing" how to move these objects from a threat mitigation standpoint; developing the capability to alter the orbits of comets and asteroids routinely for nondefensive purposes could greatly increase the probability that we can successfully divert a future impactor. The vision for CAPS is primarily to provide planetary defense, and to provide productive science, resource utilization and technology development when the system is not needed for the infrequent diversion of impacting comets and asteroids.

This paper provides a summary of CAPS and discusses several of the key areas that are being investigated: precision orbit determination, optical interferometry, and rapid rendezvous with laser ablation for orbit modification. The paper concludes with a discussion of some key technologies that would permit a permanent, continuous detection system, and an orbit modification system capable of altering the orbits of asteroids and comets in a controlled, rapid manner.

\section{BACKGROUND}

While many aspects of the impact hazard can be addressed using terrestrial-based telescopes, the 
$5^{\text {rd }}$ International Astronautical Congress IAC-02-IAA.13.4./Q.5.1.01

ability to discover and track faint and/or small comets and asteroids is tremendously enhanced, if not enabled, from space. Just as the Hubble Space Telescope has expanded our ability to see the universe without the limitations imposed by the Earth's atmosphere, a space-based NEO detection system would allow us to expand the range of comets and asteroids that we can observe, and provide coordinated follow-up observations.

A space-based detection system is capable of making observations on a continuous basis, without the various constraints (daylight, weather, etc.) imposed on Earth-based systems, and NEO searches need not be focused on the solar opposition point. If detection systems can be designed to observe faint NEOs that appear to be near the Sun, which is impossible from the ground because the atmosphere scatters sunlight during the daytime, it would be possible to see objects close to the Sun, and on the solar far side where solar illumination conditions are favorable. Additionally, it is critical to ascertain, to the greatest extent possible, the composition and physical characteristics of these objects. A space-based approach can solve this aspect of the problem, both through remote observations and rendezvous missions with the NEO. Finally, any attempt to deflect an impacting NEO with any reasonable leadtime is likely only to be accomplished using a spacebased deflection system.

It is recognized, and appreciated, that the currently funded terrestrial-based detection efforts are a vital and logical first step. Focusing on the detection of large asteroids capable of global destruction is the best expenditure of limited resources. However, various aspects of the impact threat are largely unaddressed by these efforts. There is currently no specific search for LPCs, small NEAs, or small SPCs. Additionally, coordinated follow-up observations are critical to limit the likelihood of losing a newly discovered NEO, and to determine the object's orbit. One short coming of current ground-based efforts is the difficulty in providing these follow-up measurements, which are in part provided by amateur astronomers. Looking for much smaller and fainter targets is likely to exceed the capabilities of many asteroid and comet "hunters" on Earth.
October 10-19, 2002

Houston, Texas USA

It is likely that the next object to impact the Earth will be a small near-Earth asteroid or comet. The most significant danger from smaller NEOs (several hundred meters in diameter) may result from ocean impacts, which can generate tsunamis capable of massive destruction on distant shorelines. A globally devastating impact with a $1 \mathrm{~km}$ class LPC will not be known decades, or even years, in advance with our current detection efforts. Searching for, and protecting ourselves against these types of impactors is a worthwhile endeavor. A space-based detection system, despite being more costly and complex than Earth-based initiatives, is the most promising way of expanding the range of objects that could be detected, and surveying the entire celestial sky on a regular basis. Current ground-based efforts should be expanded, and a coordinated space-based system should be defined and implemented. CAPS is an attempt to begin the definition of that future spacebased system, and identify the technology development areas that are needed to enable its implementation.

\section{CONCEPT OVERVIEW}

The combination of the words "comet" and "asteroid" in the CAPS acronym is intended to convey the idea of utilizing a combined approach for protection against both types of these cosmic projectiles. Conventional ground-based telescopes may provide little or no advanced warning of a collision with a small NEA or LPC, and developing and maintaining separate space-based systems may be impractical. Precise orbit determination of most NEAs and SPCs can be expected to be obtained several orbital periods prior to a collision, provided that we actually have the ability to observe them. This would not be the case for impacting LPCs, whose orbits need to be characterized very accurately over a small observation arc on their first observed perihelion passage through the solar system. If the situation does occur in which a small NEA is first detected on its final approach, mitigation of the impact may prove problematic. In this case CAPS would at least provide an accurate assessment of where the object would impact, and enough warning time to allow some appropriate civil defense effort to be carried out successfully. 
$5^{\text {rd }}$ International Astronautical Congress IAC-02-IAA.13.4./Q.5.1.01

The timely detection of LPCs, even those of significant size, presents many intractable problems. LPCs can be extremely faint (albedos of $\sim 0.02$ ) until the sublimation of their volatile frozen gases begins. Moreover, comets can remain in a dormant state during their perihelion passage, or they can exhaust their volatiles and become extinct nuclei. Observing LPCs at significant distances from the Sun is a formidable task. The ability to predict their orbits accurately, and hence determine whether or not they represent a threat, is dependent upon the number, resolution, and spacing of observations of these objects. Finally, the comet's trajectory can be significantly altered by non-gravitational forces if it becomes active, affecting our ability to predict its path and properly alter it. The ability to observe faint LPCs and rapidly determine their orbits is consistent with protection against small, previously undiscovered NEAs. A system capable of protecting against LPCs, placed properly in heliocentric space, should also be capable of protecting against small NEAs and small SPCs.

The baseline detection concept advocates the use of high-resolution telescopes with advanced detector arrays, coordinated telescope control for $\mathrm{NEO}$ surveying and tracking, rapid spectral imaging for NEO identification and characterization, and interferometric techniques to obtain precision orbit determination when required. Detection telescopes would be orbiting and/or lunar surface based, providing surveys of nearly the entire celestial sky approximately every 30 days. Orbiting telescopes could be placed in heliocentric orbits, including Earth-Sun libration points, or around a planetary body or moon. The CAPS detection system would provide a high probability that impacting NEOs are detected, and their orbits accurately characterized with significant warning time, even upon their first observed near-Earth approach.

The primary orbit modification approach uses a spacecraft that combines a multi-megawatt electrical power system, a high thrust and specific impulse propulsion system for rapid rendezvous, and a pulsed laser ablation payload for changing the target's orbit. This combination of technologies may offer a future orbit modification system that could deflect impactors of various compositions, and provide an effective method for altering the orbits of NEOs for
October 10-19, 2002

Houston, Texas USA

resource utilization. We envision a future for mankind in which asteroids and cometary bodies are routinely moved to processing facilities, with a permanent infrastructure that is capable and prepared to divert those objects that are a hazard.

\section{$\underline{\text { Detection }}$}

It is worthwhile to describe what is meant by "detection" in relation to the CAPS detection system. Detection includes initial discovery, follow-up observations, precision orbit determination, and physical characterization. Although all aspects of the detection problem are critical, for objects with a very limited observational period the accurate assessment of their trajectory is vital.

The initial benchmark for the CAPS detection system is to be able to identify an impactor with a diameter of $1 \mathrm{~km}$ or greater, at a distance of at least 5 Astronomical Units (AU) from Earth ( $1 \mathrm{AU} \approx 150$ million $\mathrm{km}$ ), and objects as small as $50 \mathrm{~m}$ in diameter at a distance of 0.2 AU from Earth. In general, these distance limits would provide warning times of approximately one year for a $1 \mathrm{~km} \mathrm{LPC}$, and a few weeks to approximately a month for a small NEA that has not been previously cataloged. A system possessing the sensitivity to observe $1 \mathrm{~km}$ objects at $5 \mathrm{AU}$ would be capable of detecting many $50 \mathrm{~m}$ class asteroids significantly farther away than $0.2 \mathrm{AU}$, so the warning times for uncataloged NEAs could be significantly longer. Ultimately, the ability to identify a LPC on an impact course at a distance of $5 \mathrm{AU}$ from the Earth may not provide sufficient warning time, and observing further out may be valuable. Conversely, due to the unpredictable nature of comets, both with respect to their orbits and structural integrity, it may not be prudent to take any defensive action until the object is much closer. The threat of impact may change significantly if the comet becomes active, or if it fragments into a number of sizable objects. The extremely short warning times for LPCs, the large changes in orbital velocity required to avert an impact, and the orbital and compositional uncertainties make this aspect of the impact hazard particularly difficult to solve.

The envisioned CAPS detection system would feature large aperture ( $\geq 3$ meters), high-resolution telescopes capable of imaging in the ultraviolet, 
$5^{\text {rd }}{ }^{\text {International Astronautical Congress }}$ IAC-02-IAA.13.4./Q.5.1.01

optical, and infrared wavelengths. Coordinated telescope control for NEO surveying and tracking would be incorporated to maximize follow-up observations, and baffling and/or shading would be employed to permit observations close to the Sun. Figure 1 depicts a lunar-based option with a detection node consisting of a wide field-of-view (FOV) survey telescope located in the center, and three narrow FOV tracking telescopes (telescope enclosures and/or baffling are not shown). Two detection nodes, located in the northern and southern lunar hemispheres, could provide nearly complete sky coverage every month. Each telescope would have large area mosaic detector arrays (approximately $36 \mathrm{~K} \times 36 \mathrm{~K}$ pixels), with the survey telescopes having a $1.0 \times 1.0 \mathrm{deg}$. FOV and the tracking telescopes having a $0.1 \times 0.1 \mathrm{deg}$. FOV. Spectral imaging would be implemented as early as possible in the detection process. Advanced detectors capable of rapid identification of NEOs and their spectral signal could greatly simplify operations and minimize the requirements on the tracking telescopes. If NEOs could be uniquely identified in multiple survey images, a preliminary orbit could be determined with minimal risk of "losing" the object. The tracking telescopes would be used in an interferometric mode when higher precision astrometric observations are needed to confirm an object has an impacting trajectory. Finally, active laser ranging could be used to provide range and range-rate data to augment precision orbit determination. Active laser ranging is preferable to radar systems due to the potentially large distances

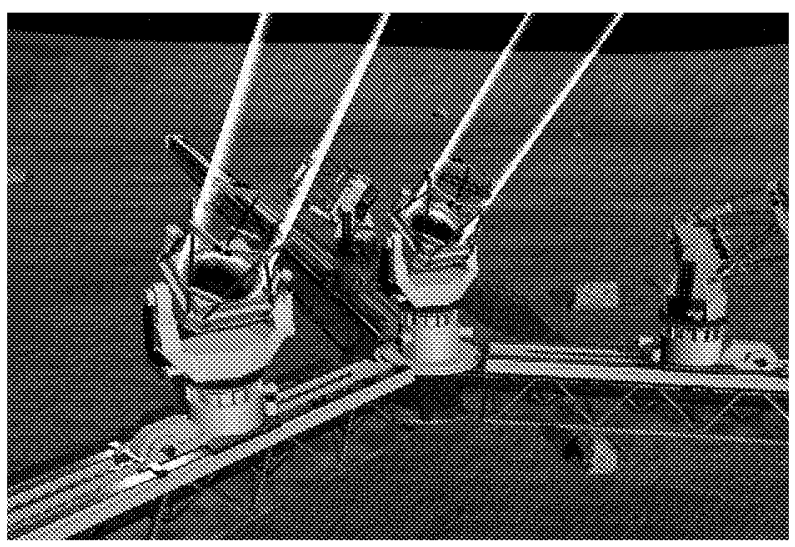

Figure 1. Depiction of Detection System Using a Lunar-Based Approach.
October 10-19, 2002

Houston, Texas USA

between the target and the detection system. The tracking telescopes could be used as receivers for the laser ranging system, or the return signal of faint NEOs could be enhanced through active illumination to aid in interferometry measurements.

\section{$\underline{\text { Deflection/Orbit Modification }}$}

Figure 2 depicts a promising, advanced method of orbit modification that combines a multi-megawatt electrical power system, a high thrust and specific impulse propulsion system for rapid rendezvous, and a pulsed laser ablation payload for changing the target's orbit. This combination of technologies may offer a future orbit modification system that could deflect an impactor and provide an effective method for altering the orbits of objects for resource utilization.

Besides rapid and controlled trajectory modification, one of the goals of CAPS orbit modification is to be effective against NEOs of various compositions. Asteroids range from primarily stony to mostly metallic, with various proportions of each type of material, and may contain deep, powdery regolith which can affect deflection efforts, particularly landing and attaching to the object. Comets contain a mixture of non-volatile materials and large amounts of frozen volatiles. When a comet becomes active, these volatiles create a diffuse cloud surrounding the nucleus called the coma. This variety of compositions and environments makes the issue of mitigation difficult, and suggests that orbit modification methods that can move the NEO without landing on it may be highly advantageous. Since time may be critical, this approach could diminish the need for detailed physical characteristic observations to be made before dispatching a deflection effort. Ultimately, a spacecraft capable of rapid interception of an incoming impactor is extremely beneficial, and several approaches for modifying the NEO's orbit could be incorporated into the deflection system. One of the most commonly cited methods for deflecting or pulverizing a threatening NEO on its final approach is the use of a nuclear detonation (Ref. [1]). However, there are many issues associated with this mitigation technique (fragmentation, radiation, etc.), and it is unlikely that the CAPS goal of controlled orbit modification can be achieved with this 
$5^{\text {rd }}$ International Astronautical Congress IAC-02-IAA.13.4./Q.5.1.01

technique. Moreover, there is a great deal of uncertainty as to how effective a nuclear explosion would be against a porous or non-monolithic object that is effectively a gravitationally bound "rubble pile" (Ref. [2]). Compatible secondary mitigation modes could be carried as an additional payload for a deflection mission, or a phased approach using rendezvous and intercept trajectories with various payloads could provide a robust defense. Regardless of the mitigation method used, rapid engagement of an object is critical for preventing an impact from a newly discovered LPC or asteroid.

It is essential to understand that the issues associated with detection and deflection of an impactor are intimately connected, particularly if we are not afforded decades of warning time that would be likely for large NEAs. The requirements for the detection system could be significantly reduced given an extremely robust deflection capability. However, due to the enormous amounts of energy required to move these massive bodies, any additional warning time is an extremely valuable asset.

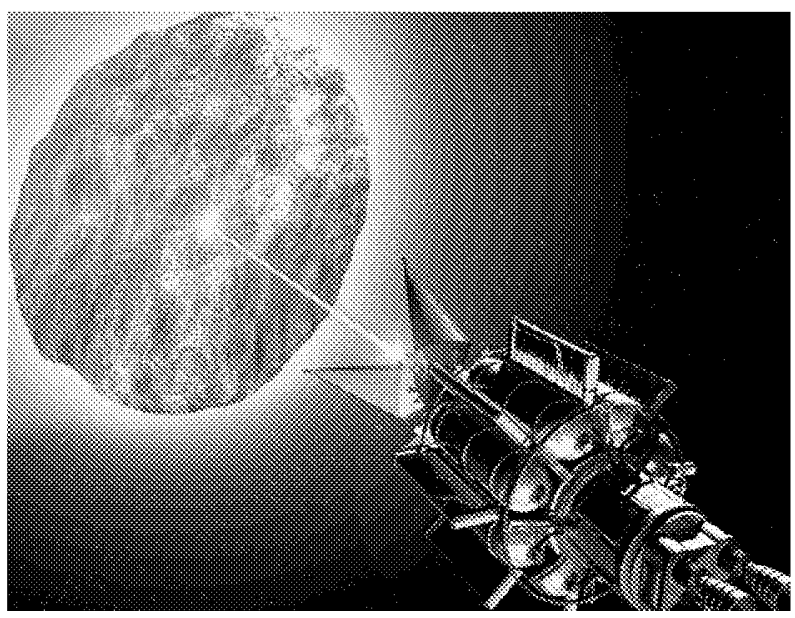

Figure 2. Depiction of Rendezvous Spacecraft with Laser Ablation Payload.

\section{ORBIT DETERMINATION}

Orbit determination is the process of using a collection of measurements obtained by observation to calculate a set of orbital elements, six quantities that give (either implicitly or explicitly) the position and velocity of an object at a particular instant of
October 10-19, 2002

Houston, Texas USA time. The quality of the result is affected by errors in the measurements, the spatial and temporal spacing of observations, the number of observations, and by the number and placement of observatories. Using the method of least squares and a batch filter (Ref. [3]), we have studied the effects of these factors in order to identify trends, and make a preliminary determination as to the number, placement, and resolution of optical instruments required to form an effective system for determining orbits of comets and asteroids.

An accurate determination of the orbit of a dangerous body is necessary in order to know if, when, and where on the Earth's surface a collision will occur. The objective of our study is to predict and then prevent such an event; therefore, the conditions required for orbital collisions are central to our analysis. For an object in heliocentric orbit with radius of perihelion $r_{p}$ and eccentricity $e$, the necessary condition for a collision in the ecliptic plane at a heliocentric distance of $r_{k}$ is that the argument of perihelion $\omega$ satisfy the relationship

$$
\pm \cos \omega=\frac{r_{p}}{r_{k}}\left(1+\frac{1}{e}\right)-\frac{1}{e}
$$

where the positive sign indicates the condition is imposed at the ascending node, and the negative sign is associated with the descending node.

Generally speaking, the longer the warning time (the interval between the time an object's orbit becomes known and the time of collision), the better the chances of being able to plan and execute action to prevent a collision. We assume a pre-perihelion collision with a warning time of less than one half the object's orbital period, and apply the time-offlight equation (see Eq. (4.2-9) of Ref. [4]) in a straightforward manner. For the cometary orbits studied, warning times range from 2 years in the case of an object with $r_{p}=1 \mathrm{AU}$ and aphelion $r_{a}=15 \mathrm{AU}$ (orbit period of 20 years), detected at a distance of 7 AU from the Sun, to 9.5 months when $r_{p}=0.1 \mathrm{AU}$ and $r_{a}=50 \times 10^{3} \mathrm{AU}$ (orbit period of $4 \times 10^{6}$ years), and the comet is detected at $5 \mathrm{AU}$. Warning time does not change appreciably as a function of $r_{a}$ in the range $1000 \leq r_{a} \leq 50 \times 10^{3} \mathrm{AU}$, and a reduction in $r_{p}$ by a factor of 10 reduces warning time by 
$5^{\text {rd }}$ International Astronautical Congress IAC-02-IAA.13.4./Q.5.1.01

approximately the same amount as a reduction of 1 AU in detection distance. Warning times for NEAs range from approximately 90 days for an asteroid in a $0.9 \times 1.4 \mathrm{AU}$ orbit detected at a heliocentric distance of $1.3 \mathrm{AU}$, to 7 days in the case of an asteroid with a $0.2 \times 3.0 \mathrm{AU}$ orbit spotted $1.1 \mathrm{AU}$ from the Sun.

Orbital elements determined on the basis of observations generally differ from the true orbital elements, which are unknown in practice but are specified in our analysis. A set of six elements produced by a batch filter can be compared to the set of true elements; however, it is more convenient to compare a single parameter if at all possible. We introduce such a parameter $\varepsilon$ called the erroneous predicted miss distance, defined to be the magnitude of the difference between an object's determined position and the specified position, evaluated at the specified time of collision. This scalar metric allows us to choose easily between two observatory configurations that have obtained different sets of measurements.

A single hypothetical cometary orbit is used to study how the accuracy of preliminary orbit determination based on three observations, each involving two measurements of angular position, is affected by the length of time of the data arc, the distance at which the object is detected, and the resolution of the telescope. A single observatory (not necessarily coincident with the Earth) is assumed to travel in the ecliptic plane in a circular orbit of radius 1 AU. Each of the six measurements is regarded as the sum of a true angle and an error produced with a pseudorandom number algorithm, uniformly distributed between the limits of the telescope's angular resolution, $\pm \rho$. In a Monte-Carlo approach, an orbit is determined 100 times using a different random number seed in each trial, and an average erroneous predicted miss distance $\bar{\varepsilon}$ is recorded. The length of the data are is varied between 10 and 98 days, heliocentric detection distance takes on the values of 5,6 , and $7 \mathrm{AU}$, and the resolution $\rho$ takes on the values $0.05,0.10$, and 0.20 arcsec (seconds of arc). With $\rho$ fixed at 0.2 arcsec, Figure 3 shows how the accuracy of orbit determination as measured by $\bar{\varepsilon}$ becomes better as detection distance decreases, with the greatest improvement occurring at the smallest data arcs; however, the most dramatic improvements
October 10-19, 2002

Houston, Texas USA

are obtained by increasing the data arc to 98 days, at which point all three values of $\bar{\varepsilon}$ are less than 2 lunar distances (1 lunar distance $=384,400 \mathrm{~km}$ ). With detection distance held fixed at $6 \mathrm{AU}$, telescope resolution is seen to have a marked effect on accuracy, especially for short data arcs, but the length of the data arc is again the most important factor and all three values of $\bar{\varepsilon}$ are below 1 lunar distance after 98 days. Unfortunately, longer data arcs yield shorter warning times.

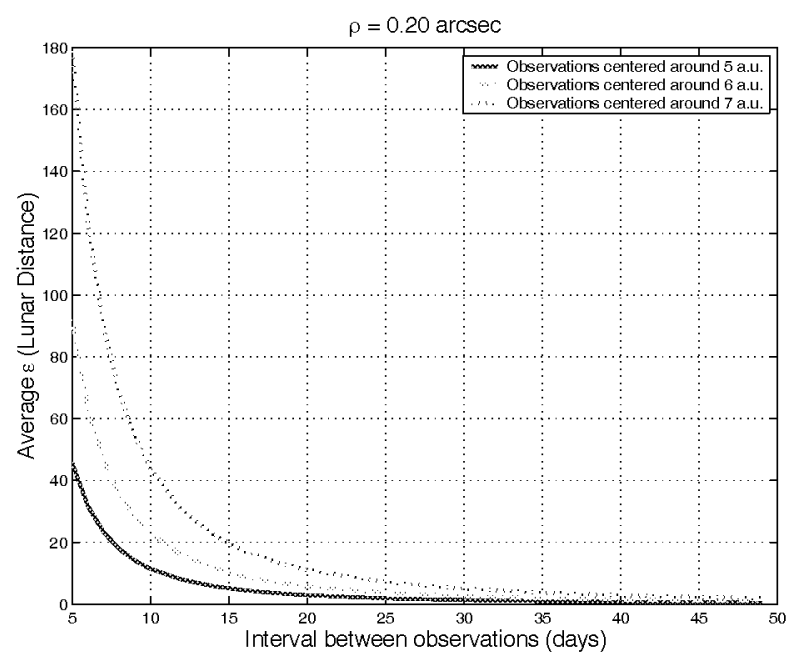

Figure 3. Average $\varepsilon$ for Various Detection Distances.

A similar approach is used in an extensive analysis of preliminary determination of long-period comet orbits. The data arc is fixed at 66 days, heliocentric detection distance is about $6 \mathrm{AU}$, and a resolution of 0.1 arcsec is assumed. Hypothetical orbits resulting in collision are constructed for 1,008 objects. A single observatory is again given a circular heliocentric orbit of radius $1 \mathrm{AU}$ in the ecliptic. The effect of observatory position is analyzed by allowing the initial true longitude $\mathrm{L}_{0}$ of the observatory to take on 4 values, each differing by $90^{\circ}$. Thus, a total of $4 \times 1008=4032$ cases are examined. When $\bar{\varepsilon}$ is shown as a function of inclination $i$, one observes that the best orbit determination is obtained when the orbit planes of the observatory and comet are perpendicular, whereas nearly coplanar orbits yield the poorest results. This relationship to inclination stems from the fact that, with three observations, an orbit is unobservable (can not be determined uniquely) when 
$5^{\text {rd }}$ International Astronautical Congress IAC-02-IAA.13.4./Q.5.1.01

it is coplanar with the observatory's orbit. With $r_{p}=$ $1 \mathrm{AU}$, retrograde orbits are harder to determine accurately than prograde orbits. Accuracy with $r_{p}=$ $0.1 \mathrm{AU}$ is noticeably poorer than with $r_{p}=0.4$ or 0.7 $\mathrm{AU}$, and even poorer with $r_{p}=1 \mathrm{AU}$. A decrease in $r_{a}$ is associated with a decrease in orbit determination accuracy. The 4032 values of $\bar{\varepsilon}$ are sorted from largest to smallest, the worst and best cases are identified, and the effects of data arc length and $\mathrm{L}_{0}$ are then studied for these two cases. Observatory location has a significant effect on accuracy, particularly in combination with short data arcs, but the effect becomes less pronounced for data arcs longer than about 70 days. In the worst case, $\bar{\varepsilon}$ is less than 4 lunar distances (for all 4 values of $\mathrm{L}_{0}$ ) after 98 days, whereas in the best case $\bar{\varepsilon}$ is better than 0.1 lunar distance, or about 6 times the Earth's radius.

Our results involving preliminary orbit determination corroborate statements made in Refs. [5] and [6], pointing out that the length of the data arc is the single most important factor in determining the accuracy of the orbit solution. The number and precision of the measurements, the object's proximity to the observatory when the measurements are obtained, and even the use of radar measurements, are all secondary to the length of the data arc.

Improved orbit determination with multiple observations is studied for the worst and best case comets. A number of observations, ranging from 3 to 99 , are taken in equally timed increments over a period of 98 days, and a resolution of 0.1 arcsec is assumed. With a single observatory, $\bar{\varepsilon}$ is reduced in the worst case from 3.8 to 0.22 lunar distances, or about 13 Earth radii. In the best case, $\bar{\varepsilon}$ is reduced from 2.5 Earth radii to 0.5 Earth radius. The improvement is pronounced for the lower numbers of observations, and gradual for the higher numbers. Further improvements afforded by measurements made with a mixture of resolutions are examined; $\rho=0.01$ aresec for initial observations and $\rho=$ 0.0001 arcsec for some number of final observations, where the total number of observations is 11 . Using the method of weighted least squares, $\bar{\varepsilon}$ is reduced in the worst case from 3.5 Earth radii when all 11 observations are obtained at the poorer resolution, to 0.25 Earth radius when the final 6 observations
October 10-19, 2002

Houston, Texas USA

employ the better resolution, as shown in Fig. 4. In the best case, $\bar{\varepsilon}$ is reduced from 0.14 to less than 0.01 Earth radius.

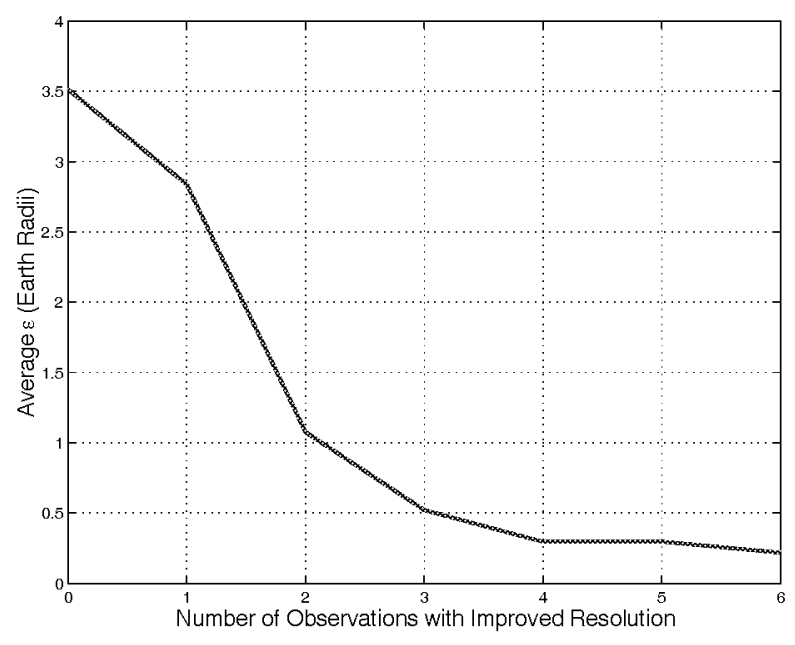

Figure 4. Observations with Mixed Resolutions for Worst Case Orbit.

The benefits of configurations with 2,3 , and 4 observatories $(\rho=0.1$ arcsec) are studied with the aid of the worst and best cases. The positions of two observatories are constructed such that they have heliocentric circular orbits of radius $\mathrm{l} A \mathrm{AU}$ in the ecliptic plane, and their true longitudes $\mathrm{L}_{0}$ are always $180^{\circ}$ apart. Three observatories are placed in similar orbits, with their true longitudes phased by $120^{\circ}$. In the case of four observatories, the first two have orbits identical to the configuration of two observatories just described, and the remaining two are in similar coplanar orbits perpendicular to the ecliptic with the ascending node $\Omega$ taking on values of $0^{\circ}, 45^{\circ}$, and $90^{\circ}$ in order to determine what effect, if any, $\Omega$ has on orbit determination. In connection with the worst comet orbit, two or more observatories offer an improvement in $\bar{\varepsilon}$ of nearly a factor of 10 over that from a single observatory. With 99 observations each, two observatories yield $\bar{\varepsilon}$ less than 0.016 lunar distance, slightly less than 1 Earth radius. Three observatories are only marginally better than two observatories. Four observatories are not substantially better than three, and $\bar{\varepsilon}$ is relatively insensitive to the value of $\Omega$ for the members of the four-observatory configuration that have orbits normal to the ecliptic. Similarly, in the best case substantial improvement in $\bar{\varepsilon}$ is 
$5^{\text {rd }}$ International Astronautical Congress IAC-02-IAA.13.4./Q.5.1.01

obtained by employing two observatories phased by $180^{\circ}$ instead of a single observatory, but the addition of a third or fourth observatory does not appear to provide any significant improvement in $\bar{\varepsilon}$.

Large data arcs are unlikely to be available for objects on a final approach, in which case the frequency and resolution of the observations become very important. Significantly improving the resolution of a single observatory would likely require the telescope diameter to become excessive. Every order of magnitude decrease in the resolution requires a telescope with a diameter 10 times larger. Optical interferometry is another approach to improving the resolution by combining the light from multiple telescopes of a more moderate size. Multiple observatories widely spaced in heliocentric space are unlikely to lend themselves to this advanced observational technique because of overresolution and disappearance of fringe patterns. If multiple telescopes at a single location can be combined into an interferometric system, the effective system resolution can be greatly increased when needed for precise orbit determination of a particular object.

Using a Kalman filter, which allows observations to be incorporated into the orbit determination solution as they become available, the worst case comet orbit has been analyzed using observations from a single observatory with various angular resolutions to demonstrate how improved resolution results in longer warning times. Figure 5 shows the amount of time required to reduce $\bar{\varepsilon}$ to 1 lunar distance, and then 1 Earth radius, assuming all observations are possible. The ultimate purpose of the CAPS detection system is to maximize warning time, which we can now take to be the difference between the time until collision ( 747 days in this case), and the time required to reduce $\bar{\varepsilon}$ to 1 Earth radius or less. In this comparison, one observation is taken every 7 days from a single observatory with angular resolutions of 0.1 or 0.01 arcsec until $\bar{\varepsilon}$ is reduced to 1 lunar distance. At this time, the frequency of observations is increased to once per day. It takes approximately 300 days to reduce $\bar{\varepsilon}$ to 1 Earth radius with $\rho=0.1$ arcsec, and 140 days with $\rho=$ 0.01 arcsec. If multiple telescopes with an initial resolution of 0.01 arcsec can provide an effective interferometric resolution of $0.0001 \mathrm{arcsec}$, an
October 10-19, 2002

Houston, Texas USA

accuracy of 1 Earth radius is achieved in only 110 days.

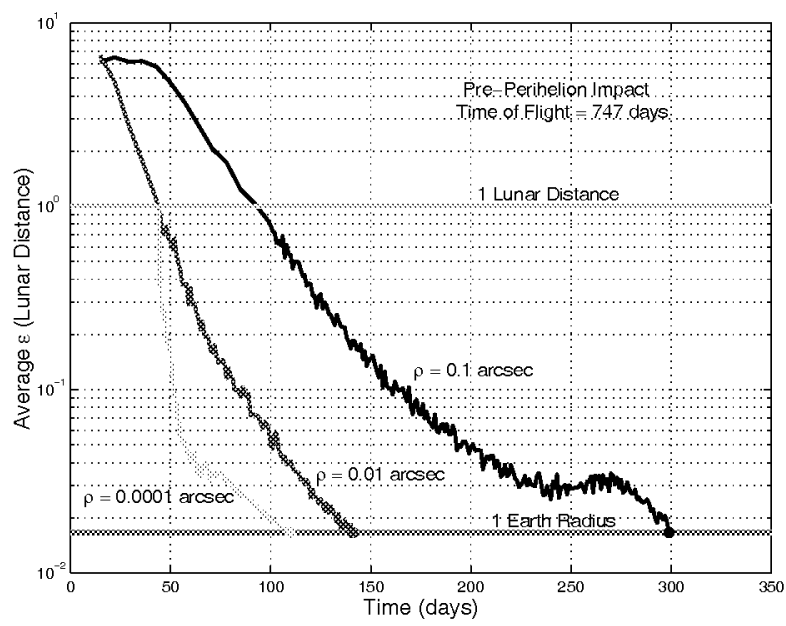

Figure 5. Improvement in Warning Time Resulting from Improvement in Angular Resolution.

We have examined 180 orbits of hypothetical NEAs of the interior kind with $r_{p}<1 \mathrm{AU}$ and $r_{\alpha}=1 \mathrm{AU}$, as well as 1350 orbits of exterior NEAs with $r_{p} \leq 1 \mathrm{AU}$ and $r_{a}>1 \mathrm{AU}$. The focus of the analysis is on asteroids discovered less than one orbit period prior to collision. Preliminary orbit determination is performed with 4 observations of 0.1 arcsec resolution taken over a 33 day data arc ending 16 days before collision. Initial observatory true longitude is again varied in increments of $90^{\circ}$, resulting in a total of 6120 cases involving asteroids. Of the 720 interior cases, 697 values of $\bar{\varepsilon}$ are less than 0.05 lunar distance, or about 3 Earth radii. Accuracy suffers for orbits with low inclinations, which is not unexpected because orbits that are coplanar with the observatory require a minimum of 4 observations if they are to be determined uniquely. Of the 5400 exterior cases, 5389 yielded $\bar{\varepsilon}$ less than 1 lunar distance. Relatively poor orbit determination is exhibited once again for orbits with low inclinations. Accuracy generally decreases for smaller values of $r_{p}$, and for larger values of $r_{\alpha}$. With each type of asteroid, 9 cases contained some number of trials out of 100 that exhibited convergence problems.

The results indicate that, as long as the measurements are available, it may be possible to 
$5^{\text {rd }}$ International Astronautical Congress IAC-02-IAA.13.4./Q.5.1.01

make reliable forecasts with two observatories whose angular resolution is on the order of 0.1 arcsec, or with a single observatory whose resolution is better by 1 to 3 orders of magnitude. Additional study of these two alternatives will have to weigh the advantages in geometry and redundancy of multiple observatories against the expense of putting them in place and maintaining them; a single observatory near Earth could be easier to maintain, but two widely spaced observatories may offer observational advantages, particularly for LPCs.

\section{NEO ASTROMETRIC INTERFEROMETRY}

Increasing the angular resolution for measurements of NEOs increases the precision of determining their relative position in the sky which, in turn, decreases the error in the orbit determination. Analysis shows that the relationship between resolution and $\bar{\varepsilon}$ is approximately linear, where dividing the resolution by two, for example from 0.2 to 0.1 arcsec, will result in a decrease in $\bar{\varepsilon}$ by a factor of two. Also, improving the resolution can result in significantly more warning time, increasing the likelihood that mitigation efforts will be successful. Therefore, any method that can provide higher resolution angular measurements of NEOs will be beneficial to orbit determination, especially when observational time is limited. Astrometric interferometry is one method that has the potential to improve angular resolution by several orders of magnitude.

\section{Theory}

Interferometry is the measurement of interference fringes produced by combining the light from two or more telescopes that observe the same source. Figure 6 shows the basic setup of an interferometer. The vector between the two telescopes is known as the baseline $\mathbf{B}$. The unit vector from the centerline of the interferometer to the source being observed is called the position vector $\mathbf{S}$. The observable in astrometric interferometry is called the delay D. Since the target being observed does not usually lie on the perpendicular axis of the interferometer, the light from the source will reach one telescope before the other as shown in Fig. 6. In order to interfere the same photon, a delay line must be introduced in the telescope that receives the photon first. As the delay line is varied, the interferometer measures an
October 10-19, 2002

Houston, Texas USA

intensity pattern shown in Fig. 7. The position of the internal delay line that gives the maximum intensity is equal to the external path delay and determines the value of $D$. This delay position is then used with the equation

$$
\mathrm{D}=|\mathbf{B}| \cos \theta
$$

to solve for $\theta$, which gives the angle between the object pointing vector $\mathbf{S}$ and the baseline orientation vector B. The baseline vector orientation in inertial

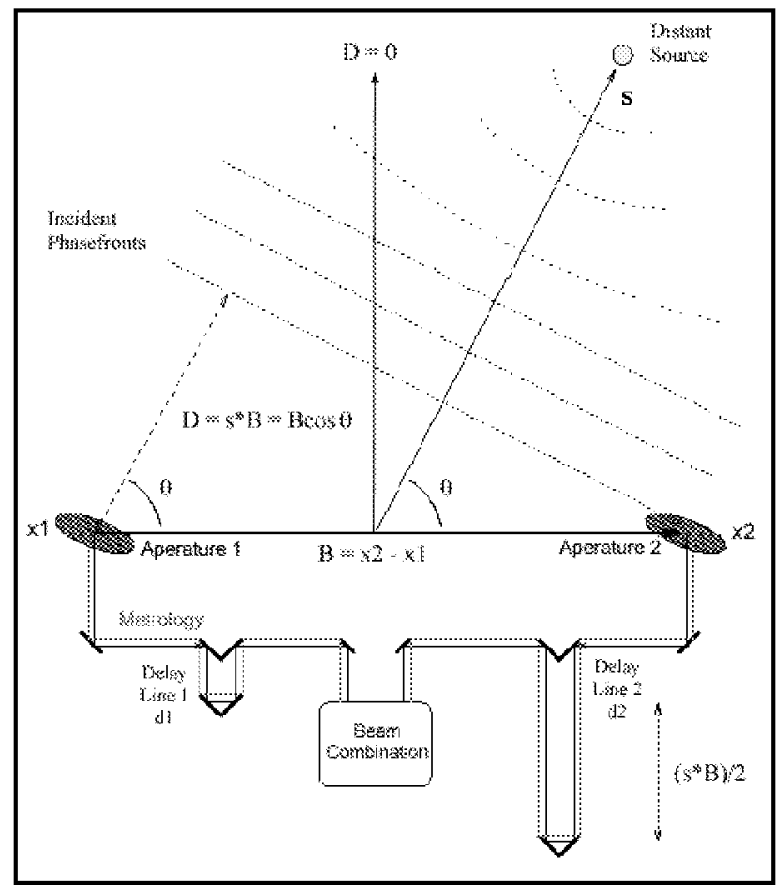

Figure 6. Basic Interferometry Layout.

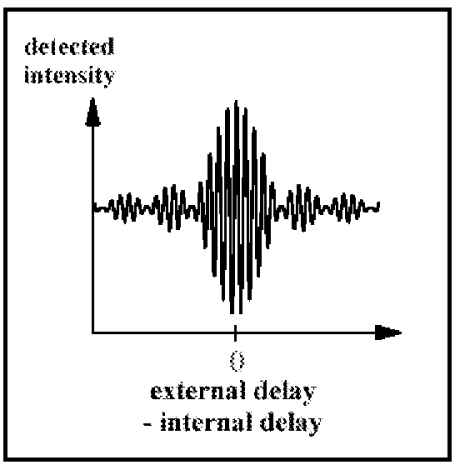

Figure 7. Interferometric Intensity Pattern. 
$5^{\text {rd }}$ International Astronautical Congress IAC-02-IAA.13.4./Q.5.1.01

space is found by observing guide stars whose positions are very well known. The baseline length and the delay position are measured with an internal metrology system. Two measurements at different baseline orientations ( $\sim$ orthogonal) are required to derive the target's two-angle position.

\section{Performance}

The angular precision of an interferometer is determined by two main factors: baseline length and Signal-to-Noise Ratio (SNR). The angular resolution of an interferometer, also known as the fringe spacing (FS), is given by the equation

$$
\mathrm{FS}=\frac{\lambda}{\mathrm{B}}
$$

where $\lambda$ is the average wavelength being observed and $B$ is the baseline length (the magnitude of $\mathbf{B}$ ). However, if SNR is high enough, measurements of even higher precision $\mathrm{P}$ can be obtained; the approximate relationship is

$$
\mathrm{P} \approx \frac{\mathrm{FS}}{\mathrm{SNR}}
$$

where $\mathrm{P}$ can also be referred to as the photon-noiselimited accuracy of the interferometer (Ref. [7]). This improvement in precision, over the theoretical angular resolution, is analogous to blur centroiding in a conventional telescope: the higher the SNR, the more accurately the center of the maximum interference fringe can be determined. Therefore, the longer the baseline and the higher the SNR, the more precise interferometry becomes. However, there is a limit to the maximum baseline that can be used to observe NEOs. As the baseline is increased, the NEO will become over-resolved, reducing the fringe visibility $\mathrm{V}$, and the fringe pattern will eventually disappear. The maximum allowable baseline depends on the object's diameter and its distance from the detector. Table 1 lists the approximate maximum baseline distance that will give measurable fringes for a $1 \mathrm{~km} \mathrm{LPC} \mathrm{and} \mathrm{a} 50 \mathrm{~m}$ NEA at their expected observational range. To detect and track the fringes, a minimum SNR of 5 is required (Ref. [7]). Using a value of $750 \mathrm{~nm}$ for the average wavelength, and a conservative SNR of 7 ,
October 10-19, 2002

Houston, Texas USA

minimum estimates of resolution are calculated for each baseline listed in Table 1.

\section{$\underline{\text { Limitations }}$}

Achieving the minimum SNR of 7 will be the most difficult part of using interferometric techniques for $\mathrm{NEO}$ astrometry. The only light that comes from a comet or asteroid is the light it reflects from the Sun. Table 2 shows expected values of visual magnitude, $\mathrm{V}_{\mathrm{m}}$, based on albedos of 0.02 and 0.154 . At a given distance from the detector, the maximum and minimum visual magnitude values will vary based on the heliocentric positions of the NEO and the detector. Note that a larger visual magnitude indicates a fainter celestial object.

For an interferometer, the SNR per pixel can be approximated by the following equation (Ref. [8])

$$
\mathrm{SNR} \approx \frac{R_{n} c}{B \sigma_{\dot{\theta}} r \sqrt{2 v \Delta v}} V
$$

where $R_{n}$ is the photon rate per pixel, $c$ is the speed of light, $V$ is the fringe visibility, $B$ is the magnitude of the baseline vector, $\sigma_{\dot{\theta}}$ is the uncertainty in angular rates, $r$ is the read noise, and $v \Delta v$ is the passband.

Four important factors that limit the SNR for LPCs and NEAs are the photon rate, uncertainty in angular rates, fringe visibility, and read noise. The photon rate is related to the visual magnitude of the object. Since many NEOs are intrinsically very faint, the only way to increase the photon rate is by using larger aperture telescopes. The uncertainty in the angular rate is due to the object's motion in the solar system as well as disturbances in the interferometer itself. Since only an estimated trajectory of the object will be available, there will be some angular rate error as the estimated trajectory deviates from the actual trajectory during the observing time. This value is analogous to integration time. The less deviation in the estimated path, the longer the integration time for a particular measurement. Although the long-term deviation in the object's orbit is uncertain, the short-term variations are expected to be relatively small. Fringe visibility is 


\begin{tabular}{|c|c|c|c|c|c|}
\hline \multicolumn{3}{|c|}{1 km Long-Period Comet } & \multicolumn{3}{c|}{ 50 m Near Earth Asteroid } \\
\hline $\begin{array}{l}\text { Distance From } \\
\text { Detector (AU) }\end{array}$ & $\begin{array}{l}\text { Maximum } \\
\text { Baseline (m) }\end{array}$ & $\begin{array}{l}\text { Estimated Angular } \\
\text { Resolution (arc-s) }\end{array}$ & $\begin{array}{l}\text { Distance From } \\
\text { Detector (AU) }\end{array}$ & $\begin{array}{l}\text { Maximum } \\
\text { Baseline (m) }\end{array}$ & $\begin{array}{l}\text { Estimated Angular } \\
\text { Resolution (arc-s) }\end{array}$ \\
\hline 6 & 168 & $1.3 \times 10^{-4}$ & 0.8 & 450 & $4.9 \times 10^{-5}$ \\
\hline 5 & 140 & $1.6 \times 10^{-4}$ & 0.6 & 337 & $6.6 \times 10^{-5}$ \\
\hline 4 & 112 & $2.0 \times 10^{-4}$ & 0.4 & 225 & $9.9 \times 10^{-5}$ \\
\hline 3 & 84 & $2.6 \times 10^{-4}$ & 0.2 & 113 & $2.0 \times 10^{-4}$ \\
\hline
\end{tabular}

Table 1. Estimated Optical Interferometry Angular Resolutions.

\begin{tabular}{|c|c|c|c|c|c|c|c|c|c|}
\hline \multicolumn{5}{|c|}{$1 \mathrm{~km}$ Long-Period Comet } & \multicolumn{5}{|c|}{$50 \mathrm{~m}$ Near Earth Asteroid } \\
\hline \multirow{2}{*}{$\begin{array}{c}\text { Distance } \\
\text { From } \\
\text { Detector }(A U)\end{array}$} & \multicolumn{2}{|c|}{ Min. $V_{m}$} & \multicolumn{2}{|c|}{ Max. $V_{m}$} & \multirow{2}{*}{$\begin{array}{c}\text { Distance } \\
\text { From } \\
\text { Detector (AU) }\end{array}$} & \multicolumn{2}{|c|}{ Min. $V_{m}$} & \multicolumn{2}{|c|}{ Max. $V_{m}$} \\
\hline & $\begin{array}{c}\text { Albedo } \\
0.02\end{array}$ & $\begin{array}{c}\text { Albedo } \\
0.154\end{array}$ & $\begin{array}{l}\text { Albedo } \\
0.02\end{array}$ & $\begin{array}{c}\text { Albedo } \\
0.154\end{array}$ & & $\begin{array}{l}\text { Albedo } \\
0.02\end{array}$ & $\begin{array}{c}\text { Albedo } \\
0.154\end{array}$ & $\begin{array}{c}\text { Albedo } \\
0.02\end{array}$ & $\begin{array}{c}\text { Albedo } \\
0.154\end{array}$ \\
\hline 6 & 27.55 & 25.34 & 28.37 & 26.15 & 0.8 & 27.14 & 24.93 & 28.36 & 26.14 \\
\hline 5 & 26.72 & 24.51 & 27.67 & 25.46 & 0.6 & 26.26 & 24.05 & 31.03 & 28.81 \\
\hline 4 & 25.69 & 23.47 & 26.85 & 24.63 & 0.4 & 25.09 & 22.88 & 32.95 & 30.73 \\
\hline 3 & 24.32 & 22.11 & 25.81 & 23.60 & 0.2 & 23,25 & 21.04 & 33.85 & 31.63 \\
\hline
\end{tabular}

Table 2. Range of Expected Visual Magnitudes.

the ratio between the maximum and minimum measured intensities, and as stated above, is a function of the baseline distance and object diameter. The last factor, read noise, is due to the detector. Since the photon rate will be very low, even a small amount of read noise can affect the signal-to-noise ratio drastically.

\section{$\underline{\text { Requirements for NEOs }}$}

Internal metrology systems with sub-nanometer accuracy will be required to measure the delay position, as well as the baseline length. The orientation of the baseline must also be measured within $\sim 50$ micro-arcsec accuracy. The orientation is measured with respect to guide stars so a catalog of stars with known positions of 1 micro-arcsec accuracy must be used. Initial calculations show that using 3 meter diameter telescopes, with a read noise of 3 electrons in the detector, a baseline length of 100 meters, a fringe visibility of 0.5 , and a visual magnitude of 27 , requires a maximum error in the angular uncertainty rate of $\sim 5 \times 10^{-11} \mathrm{rad} / \mathrm{s}$. Initial orbit determination analysis has shown that after 99 days of observation, the uncertainty in the angular rate for a typical LPC is below $10^{-13} \mathrm{rad} / \mathrm{s}$. Keeping the angular rate errors of the interferometer itself below that level is another requirement. Since the
Earth's atmosphere limits the maximum visual magnitude and makes it impossible to observe extremely faint NEOs, a location where an atmosphere does not impede its performance is preferable, either orbiting in space or on the lunar surface.

\section{Orbiting vs. Lunar-Based}

Orbiting and lunar-based interferometers offer different advantages and disadvantages. They both benefit from the lack of an appreciable atmosphere, which limits the sensitivity of Earth-based observations. Since terrestrial-based interferometers are very well understood, putting one on the moon would be very similar. Having the interferometer fixed to the lunar surface keeps the baseline very stable, reducing measurement uncertainties. Additionally, thermal variations could be minimized during lunar "night," or by locating the interferometer in a crater that offers significant shadowing. Disadvantages of lunar-based interferometers include the problem of lunar dust degrading the optics, and observations are constrained by the Moon's rotation rate. In space, the interferometer can theoretically be aimed in any direction. Of course, moving the interferometer and maintaining a stable baseline poses its own set of 
$5^{\text {rd }}$ International Astronautical Congress IAC-02-IAA.13.4./Q.5.1.01

problems. This requires some sort of propulsion system, which not only consumes propellant but also introduces disturbances into the system that may take a long time to damp out. The issue of maintaining a stable platform, and the consumables required, are major issues in space-based astrometric interferometry.

\section{$\underline{\text { Conclusion }}$}

Initial analysis suggests that using astrometric interferometry on NEAs and LPCs from space is possible. A single 3 meter telescope gives a nominal diffraction limited resolution of $\sim 0.06$ arcsec. Therefore, astrometric interferometry could improve the angular resolution by at least two orders of magnitude, resulting in a commensurate drop in the orbit determination error. The extremely faint targets being pursued by the CAPS detection system pose an enormous challenge for using interferometry, but initial analysis shows that it should be possible to use astrometric inteferometry on NEAs and LPCs from space.

\section{ORBIT MODIFICATION USING RAPID RENDEZVOUS SPACECRAFT AND LASER $\underline{\text { ABLATION }}$}

One approach to alter the trajectory of the object in a highly controlled manner is to use pulsed laser ablative propulsion (Ref. [9]). A sufficiently intense laser pulse ablates the surface of the NEO by causing plasma blow off. The momentum change from a single laser pulse is very small. However, the cumulative effect is very effective because the laser can interact with the object over long periods of time. The laser ablation technique can overcome the mass penalties associated with other non-disruptive approaches since no propellant is required to generate the $\Delta \mathrm{V}$ (the material of the celestial object is the propellant source). Additionally, laser ablation is effective against a wide range of surface materials and does not require any landing or physical attachment to the object. For diverting distant asteroids and comets, the power and optical requirements of a laser ablation system on or near the Earth may be too extreme to contemplate in the next few decades. A hybrid solution would be for a spacecraft to carry a laser as a payload to a particular celestial body. The spacecraft would require an
October 10-19, 2002

Houston, Texas USA advanced propulsion system capable of rapid rendezvous with the object, and an extremely powerful electrical generator, which is likely needed for the propulsion system as well. The spacecraft would station-keep with the object at a "small" standoff distance while the laser ablation is performed.

Figure 8 shows an estimate of the typical energy required for laser ablation to deflect a given size asteroid or comet by 1 Earth radius, when preperihelion collision with Earth is considered. The required laser energy is also a function of the object's density and the required $\Delta \mathrm{V}$, which varies depending on the object's orbit and when the deflection occurs. NEAs and LPCs with typical orbits and bulk densities were used to estimate these energy values. The estimated energy is calculated assuming that the cumulative energy generated by the laser is applied as an equivalent impulsive $\Delta \mathrm{V}$ at some time before collision. Since the laser ablation occurs over a significant time period, the laser interaction must be complete prior to the time specified for each curve in order to assure that the deflection could be accomplished. Figure 9 shows a preliminary estimate of the achieved energy for a given laser power and operation period. Figures 8 and 9 can be used to estimate the nominal laser power required for a deflection mission. For example, Fig. 8 shows that approximately $2.5 \times 10^{4}$ GJ of energy is required to deflect a $0.2 \mathrm{~km}$ stony asteroid by 1 Earth radius if applied one year before collision. Figure 9 shows that a $10 \mathrm{MW}$ laser $(1000$ $\mathrm{kJ}$ pulse and $10 \mathrm{~Hz}$ laser repetition frequency) would be required to operate continuously for approximately 30 days in order to achieve this cumulative energy.

Fast space trips are important to intercept and rendezvous with an impacting asteroid or comet, particularly those not detected many years in advance. Fast trajectories can shorten space flight times, and allow orbit modification efforts to begin earlier. The earlier that the effort begins, the less change in velocity $(\Delta V)$ required to alter the object's trajectory. However, shorter trip times require more propellant to provide enough thrust if travel distance is fixed. This additional propellant mass can be a burden to the structural architecture of spacecraft. Thus, there exists an optimal balance between flight 
$5^{\text {rd }}$ International Astronautical Congress IAC-02-IAA.13.4./Q.5.1.01

time and propellant mass. Many future propulsion systems have been proposed and analyzed.

One potential propulsion approach that has been examined for a CAPS deflection capability is the Variable Specific Impulse Magnetoplasma Rocket (VASIMR). VASIMR is a high power magnetoplasma rocket that gives continuous and variable thrust at constant power (Ref. [10]). Hydrogen plasma is heated by radio frequency (RF) power to increase exhaust velocity up to $300 \mathrm{~km} / \mathrm{sec}$. The power output of the engine is kept constant, thus thrust and specific impulse, $\mathrm{I}_{\mathrm{sp}}$, are inversely related. Thrust is increased proportional to the power level.

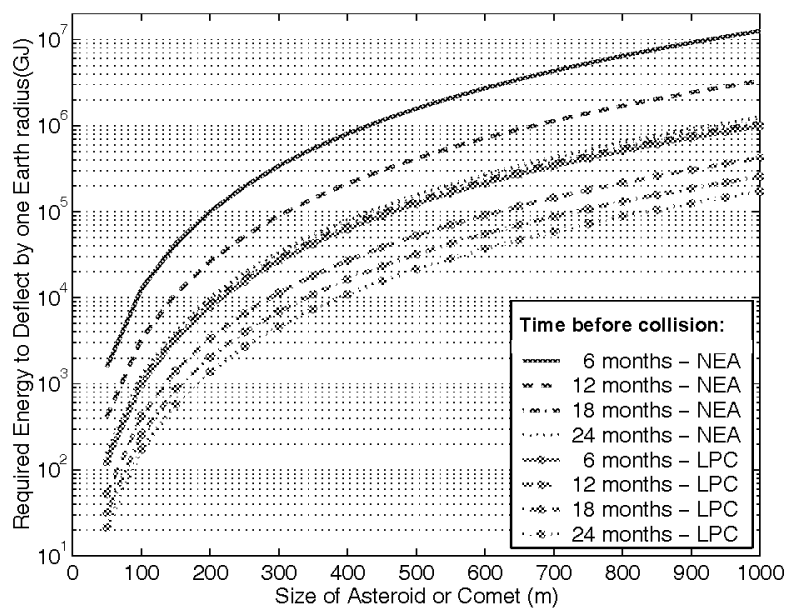

Figure 8. Estimated Energy Required for Laser Ablation vs. Diameter for Typical NEAs $\left(\rho=3000 \mathrm{~kg} / \mathrm{m}^{3}\right)$ and LPCs $\left(\rho=200 \mathrm{~kg} / \mathrm{m}^{3}\right)$.

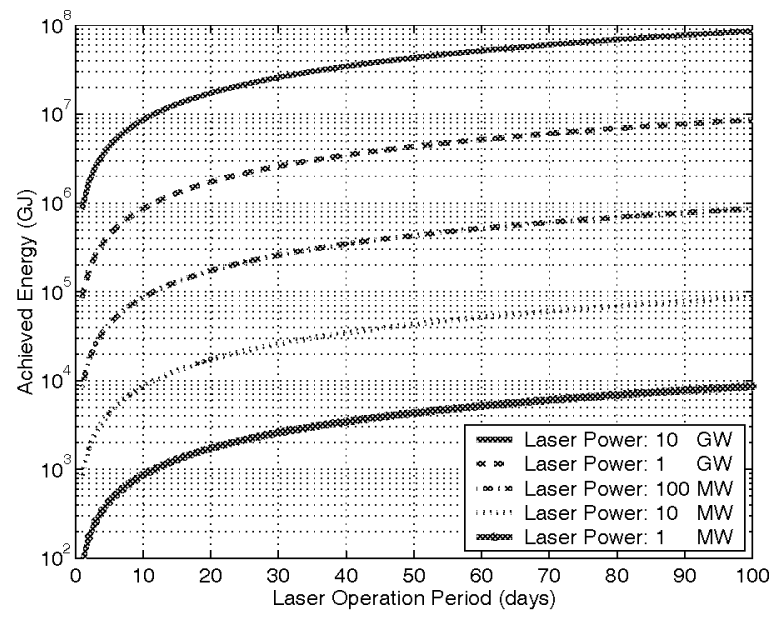

Figure 9. Estimate of Achieved Energy for a Given Laser Power and Operation Period.
October 10-19, 2002

Houston, Texas USA
The engine can optimize propellant usage and deliver a maximum payload in minimum time by varying thrust and $\mathrm{I}_{\mathrm{sp}}$ (Ref. [11]). Therefore, VASIMR can yield the fastest possible trip time with a given amount of propellant by using constant power throttling (CPT). A $10 \mathrm{~kW}$ space demonstrator experiment has been completed, and a VASIMR engine with $200 \mathrm{MW}$ power could be available around the year 2050. The specific impulse range of the engine would be $3,000 \mathrm{sec}-$ $30,000 \mathrm{sec}$, and the corresponding thrust range would be approximately $5,000 \mathrm{~N}-500 \mathrm{~N}$ (assuming $100 \%$ power efficiency).

For interception and rendezvous trajectories, optimization problems (Ref. [12]) in three dimensions are formulated to minimize flight time with moderate propellant mass. These problems minimize a weighted sum of the flight time and required propellant by using a VASIMR propulsion system with constrained specific impulse. The optimal thrust-vector history and propellant mass to use are found in order to transfer a spacecraft from the Earth to a target. For instance, we consider a fictitious impacting LPC whose orbital elements are given by semi-major axis $a=40 \mathrm{AU}$, inclination $i=$ $20^{\circ}$ and eccentricity $e=0.985$ (yields orbital period of 253 years, perihelion distance of $0.6 \mathrm{AU}$, aphelion distance of $79.4 \mathrm{AU})$. Figure 10 illustrates the trajectories and thrust vector for a 100 metric ton (mt) spacecraft (including $10 \mathrm{mt}$ payload) to intercept or rendezvous with this particular LPC, for a departure time of 8 months before a pre-perihelion collision with Earth. The propulsion system is assumed to operate at $90 \%$ power efficiency. Figures 11 and 12 show flight time and required propellant for intercept and rendezvous trajectories for various departure times, when a $100 \mathrm{mt}$ spacecraft with $200 \mathrm{MW}$ of power is assumed. For this specific LPC, there is a peak at 13 months departure time, because the spacecraft must fly in the reverse direction with respect to Earth's orbital velocity. Since there are local minima and maxima in the propellant required and flight time as shown in the figures, it can be concluded that the values are dependent upon the orbital geometry relationship as well as distance between the Earth and the comet. For a given departure time, the rendezvous trajectory requires more propellant and longer flight time than the intercept trajectory. This is because the terminal 
$5^{\text {rd }}{ }^{\text {International Astronautical Congress }}$ IAC-02-IAA.13.4./Q.5.1.01

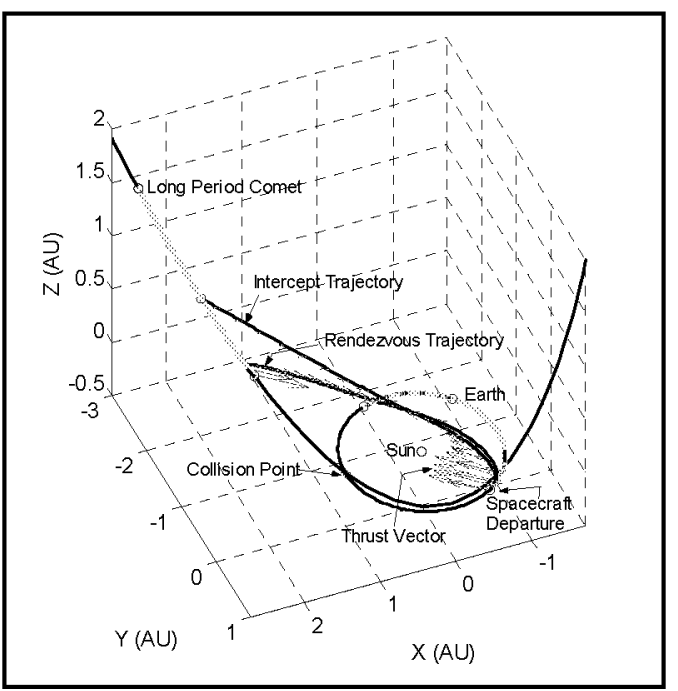

Figure 10. An Example for Intercept/Rendezvous Trajectory with Long-Period Comet.

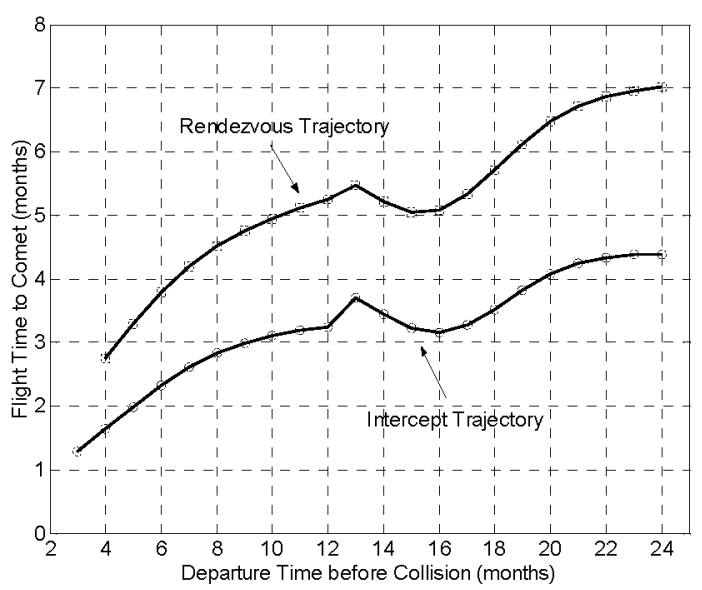

Figure 11. Flight Time for Each Departure Time.

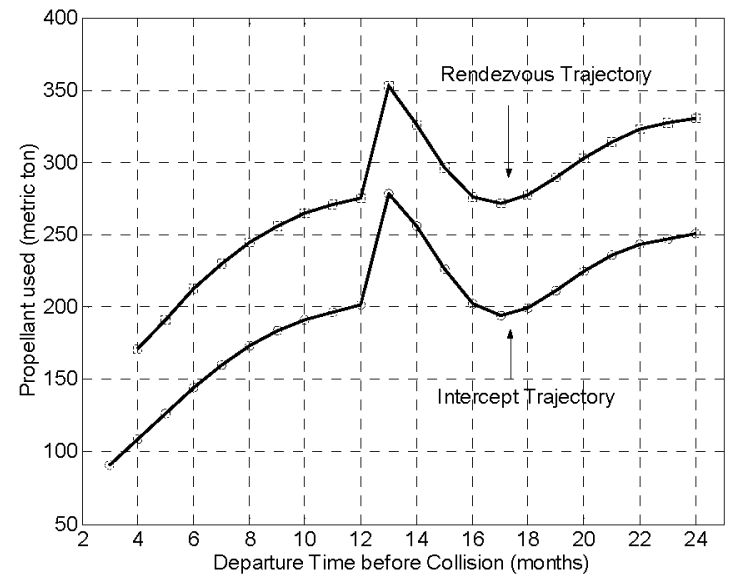

Figure 12. Propellant Used for Each Departure Time.
October 10-19, 2002

Houston, Texas USA

velocity of rendezvous spacecraft must be matched with the target's velocity, which is not required for the intercept trajectory. Asteroids and comets with different orbital elements will have different flight times and propellant requirements. Even for the same celestial object, a post-perihelion impact would have different results from those of a pre-perihelion impact.

Once the spacecraft has rendezvoused with the LPC, a laser ablation system makes use of the same multi-megawatt electrical power system that the propulsion system uses for the orbital transfer. For example, if the laser ablation operation can be completed 9 months before collision, approximately $6 \times 10^{5}$ GJ of energy would be required for a $1 \mathrm{~km}$ LPC to be deflected by 1 Earth radius. If we choose a $100 \mathrm{MW}$ laser system (assuming $200 \mathrm{MW}$ of supplied power and 50\% laser system efficiency), it would take about 70 days of continuous operation to provide this amount of energy. From Fig. 11, the rendezvous spacecraft with laser ablation system should depart from Earth approximately 16 months before the collision, with a trip time of about 5 months. For the mission, approximately $275 \mathrm{mt}$ of propellant would be required assuming $100 \mathrm{mt}$ total dry mass of the spacecraft and payload. Depending on the payload mass capability of the propulsion system, multiple mitigation devices could be delivered to the impactor, including a fall-back option to the laser ablation system. In case the rendezvous deflection mission was unsuccessful, a similar spacecraft with a different payload (e.g., nuclear explosive device) could be sent to mitigate the target using an intercept trajectory. If we assume that the intercept spacecraft departs from Earth 9 months before the collision with a $10 \mathrm{mt}$ payload, the spacecraft can arrive at the target approximately 6 months before impact and requires less than $200 \mathrm{mt}$ of propellant (as shown in Figs. 11 and 12).

A multi-megawatt nuclear electric propulsion (NEP) system utilizing a VASIMR engine is currently estimated to have a maximum overall specific mass of $1.0 \mathrm{~kg} / \mathrm{kW}$ (Ref. [13]). For a $200 \mathrm{MW}$ system, this would result in a total spacecraft dry mass of $200 \mathrm{mt}$ (neglecting payload mass). This is twice the total spacecraft mass assumed for this analysis, and the mass of a future laser ablation payload is presently not well understood. The assumed power 
$5^{\text {rd }}$ International Astronautical Congress IAC-02-IAA.13.4./Q.5.1.01

efficiency of $90 \%$ is also optimistic. More capable power generators (gigawatt class) with lower specific masses could provide the power needed to reduce trip times and provide more powerful lasers. Deflecting an impactor by only 1 Earth radius is likely to be insufficient for a deflection effort. More powerful lasers would be capable of providing a greater miss distance, and thus more margin for uncertainty in the object's orbit. NEOs with greater densities would also require a more capable laser ablation system. Longer warning times would reduce the requirements on the orbit modification system, but would make the CAPS detection system more challenging to implement. For the $1 \mathrm{~km} \mathrm{LPC}$ assumed in this analysis, the detection system would need to determine the comet's trajectory at least 16 months prior to its pre-perihelion collision with the Earth. For impactors with extremely short warning times, an intercept trajectory may be the only feasible scenario for diverting the object.

Technological advances that can significantly reduce the specific mass of the rendezvous spacecraft and laser payload may permit this type of mitigation approach to become a reality. A tiered mitigation approach using rapid rendezvous and intercept spacecraft could provide a feasible scenario to protect the Earth from an impacting LPC, as well as other classes of impacting NEOs. A rendezvous spacecraft with a laser ablation payload could provide a capable and robust orbit modification approach for altering a NEO's orbit for resource utilization.

\section{KEY TECHNOLOGIES}

The enabling technologies required for the development of a viable protection system can be divided into the two areas of detection and mitigation. Many of the detection technologies are currently in development for advanced in-space telescope systems such as the James Webb Telescope (formerly known as the Next Generation Space Telescope). Mitigation technologies are also currently being studied as part of advanced power and propulsion research. With the proper funding levels, many of the technologies needed to support a CAPS architecture could be achievable within the next 15 - 20 years. The high power, propulsion, heat
October 10-19, 2002

Houston, Texas USA

rejection, and directed energy systems would likely be farther term than some of the detection technologies.

The following are key technologies for CAPS detection capability:

- Large aperture, high resolution advanced telescopes (ultraviolet, optical, and infrared) will be required for detection as well as tracking tasks.

- Advanced lightweight mirrors could be used to reduce the launch weight of CAPS detection assets and thus reduce the overall system cost. Examples of these technologies include lowmass membrane mirror optics and liquid surface mirrors. It is likely that active control will also be required to maintain precise mirror shape.

- Large area mosaic Charge Injection Device (CID) sensor arrays (approximately $36 \mathrm{~K} \times 36 \mathrm{~K}$ pixels) are needed for rapid surveying and tracking (precise determination of target object's angular position). CIDs exhibit less light bloom from pixel to pixel when subjected to high intensity light compared to Charge Couple Device (CCD) arrays, and are also less sensitive to radiation.

- Advanced detectors capable of rapid NEO identification would be extremely valuable. The S-Cam, currently under development by the European Space Agency (ESA), uses Superconducting Tunneling Junctions (STJs), which can count individual photons and provide associated spectral information. These data could be used to detect and "tag" asteroids and comets for simplified follow-up observations, cataloging, and future identification.

- Active cooling is required to achieve optimal performance from the sensors, whether they are CCDs, CIDs or STJs, with temperature requirements being only a fraction of a degree Kelvin in the case of STJs.

- Baffling and/or shading technologies would permit observations close to the Sun (possibly within 15 degrees of the sun line), increasing the area of sky that can be sampled. The shading 
$5^{\text {rd }}$ International Astronautical Congress IAC-02-IAA.13.4./Q.5.1.01

could be in the form of an attached sunshade, a large deployable shade flying in formation with the telescope, or an internal occulting disk such as that used in particular coronagraphs.

- Optical interferometric techniques and/or laser ranging systems for precise orbit determination.

- Precision spacecraft and detector pointing will be needed to provide star field accuracy for guide stars to better than 0.001 arcsec. Accurate position and time knowledge is also needed so that the CAPS detection system can precisely acquire targets. If interferometric techniques are employed, the linear distance between two telescopes must be known to within approximately $1 \mathrm{~nm}$, and precise position determination and control with nano/pico meter knowledge must be available.

- Advanced data management systems and rapid communications will be needed for processing observation data and cataloging NEOs. Significant image data will be generated by multiple large $\mathrm{CCD} / \mathrm{CID}$ arrays from multiple telescopes potentially at remote locations. This data will have to be processed and downlinked, the resulting image data stored, and an object database created. Ultra-high data rates for downlink may be achievable using optical communications technology. Potential high bandwidth inter-satellite communications may also be needed for interferometry or database synchronization.

The following are key technologies for CAPS mitigation capability:

- High thrust, high specific impulse propulsion systems (plasma, nuclear, etc.) for delivering orbit modification systems to target NEOs.

- Multi-megawatt electrical power systems for propulsion and laser applications.

- Advanced thermal management systems to reject large amounts of waste heat.

- Reliable, high-power pulsed laser ablation systems with adaptive laser optics, precision beamwidth focusing, and closed-loop control system to provide continuous orbit modification
October 10-19, 2002

Houston, Texas USA

capability. Systems could also potentially be used as an active ranging system for precision orbit determination.

- Advanced autonomous or semi-autonomous rendezvous and station-keeping capability for engaging the NEO at close distances. Formation flying capability and precise attitude control may also be needed for interferometry using orbiting detectors.

\section{$\underline{\text { SUMMARY AND CONCLUSIONS }}$}

Many of the major issues have been identified for a futuristic capability to protect against impacting comets and asteroids, and a preliminary space-based concept has been envisioned. Some of the basic concept elements, approaches, methodologies, and features have been identified. Initial orbit determination analyses indicates that there is a tradeoff between multiple NEO observatories spread throughout the solar system and a single observatory whose resolution is better by at least an order of magnitude. Observations from heliocentrically spaced detectors offer significant geometrical advantages, and potentially allow multiple, simultaneous measurements of a NEO. However, because of their distance from Earth, they may be more difficult to deploy and maintain. When contemplating the ability to monitor comets and asteroids continuously, there are many trade-offs between orbiting observatories and detection systems on planetary bodies without an atmosphere. Interferometric techniques to provide high precision astrometric measurements appear to be feasible and may provide a vital capability to perform precise orbit determination for asteroids and comets that have only been observed for a short period of time. This is critical for protecting against long-period comets and small, uncataloged asteroids and comets. Future orbit modification techniques have the potential for rapid and controlled alteration of NEO orbits, provided that high power and compatible thermal management systems are developed.

In the future, it appears possible that even longperiod comets can be detected and deflected in time to prevent an Earth impact given sufficiently capable systems and technologies. These technologies will take years to develop, but many are important for 
$5^{\text {rd }}$ International Astronautical Congress IAC-02-IAA.13.4./Q.5.1.01

enabling for human space exploration of the solar system. Much additional work and analysis is required to identify a final system concept, and many trade studies will need to be performed to select the best mix of system capability, reliability, maintainability, and cost. Finally, it is fully appreciated that at the present time space systems are much more costly than terrestrial-based systems. Hopefully, this will change in the future. Regardless, understanding what it would take to defend against a much wider range of the impact threat will foster ideas, innovations, and technologies that could one day enable the development of such a system. This understanding is vital to provide ways of reducing the costs and quantifying the benefits that are achievable with a system like CAPS.

\section{$\underline{\text { ACKNOWLEDGEMENTS }}$}

The authors of this paper would like to recognize the efforts of the personnel and contractors at NASA Langley Research Center who have contributed to various aspects of the CAPS study under the Revolutionary Aerospace Systems Concepts (RASC) program. Special thanks are extended to Hans Seywald (Analytical Mechanics Associates, Inc.) for his technical inputs and helpful discussions. We are most grateful to Professors George Born and Steve Nerem, and Matt Hausman, all at the University of Colorado, for their guidance in the discipline of orbit determination, especially to Dr. Born who provided us with a copy of Ref. [3].

\section{REFERENCES}

[1] Canavan, G. H., Solem, J. C., and Rather, J. D. G., eds., Proceedings of the Near-Earth-Object Interception Workshop, Los Alamos National Laboratory, Los Alamos, NM, February 1993.

[2] Holsapple, K. A., "Geology of asteroids: Implication of Spin States Regarding Internal Structure and Some implications of that Structure on Mitigation Methods," Presentation at the 2002 NASA Workshop on Scientific Requirements for Mitigation of Hazardous Comets and Asteroids, Arlington, VA, Sept. 3-6 2002.

[3] Tapley, B. D., Schutz, B. E., and Born, G. H., Fundamentals of Orbit Determination, Academic Press, to be published.
October 10-19, 2002

Houston, Texas USA

[4] Bate, R. R., Mueller, D. D., and White, J. E., Fundamentals of Astrodynamics, Dover Publications, Inc., New York, 1971

[5] Chodas, P. W., and Yeomans, D. K., "Orbit Determination and Estimation of Impact Probability for Near Earth Objects," Proceedings of the $22^{\text {nd }}$ Annual AAS Rocky Mountain Guidance and Control Conference, Breckenridge, CO, Feb. 3-7, 1999 (A99-34626 09-12), San Diego, CA, Univelt, Inc. (Advances in the Astronautical Sciences. Vol. 101), 1999, pp. 21-40.

[6] Chodas, P. W., et al., "Automated Detection of Potentially Hazardous Near-Earth Encounters," AAS 01-461, AAS/AIAA Astrodynamics Specialists Conference, Quebec City, Quebec, Canada, July 30August 2, 2001.

[7] Shao, M., and Colavita, M. M., "Potential of long-baseline infrared interferometry for narrowangle astrometry," Astron. Astrophys., Vol 262, pp. 353-358, 1992.

[8] Lay, O., "Separated Spacecraft Interferometry," Presentation at the 2002 Michelson Interferometry Summer School, Harvard Smithsonian Center for Astrophysics, June 24-28, 2002.

[9] Phipps, C. R., "Lasers Can Play an Important Role in the Planetary Defense," Proceedings of the Planetary Defense Workshop, Lawrence Livermore National Laboratory, Livermore, CA, May 1995.

[10] Chang-Diaz, F. R., et al., "The Physics and Engineering of the VASIMR Engine," 36th AIAA/ASME/SAE/ASEE Joint Propulsion Conference, 17-19 July 2000, Huntsville, AL, AIAA 2000-3756.

[11] Chang-Diaz, F. R., "The VASIMR Rocket," Scientific American, November 2000, pp. 90-97.

[12] Bryson, A. E., Dynamic Optimization, Addison-Wesley, Menlo Park, CA, 1999.

[13] Smith, B., Knight, T., and Anghaie, S., "Multimegawatt NEP with Vapor Core Reator MHD," Space Technology and Applications International Forum (STAIF 2002), Albuquerque, NM, Feb. 3-6 2002. 